DOI: 10.1515/awutp -2015-0022

\title{
USING ALGEBRAIC COMPUTING TO TEACH GENERAL RELATIVITY AND COSMOLOGY
}

\author{
Dumitru N. Vulcanov, Remus-Ştefan Ş. Boată
}

West University of Timişoara, Department of Physics, B-dul V. Pârvan no. 4, 300223, Timişoara, România

\author{
Article Info \\ Received: 20 December 2011 \\ Accepted: 29 March 2012
}

Keywords: Algebraic computing, Cosmology

\begin{abstract}
The article presents some new aspects and experience on the use of computer in teaching general relativity and cosmology for undergraduate students (and not only) with some experience in computer manipulation. Some years ago certain results were reported [1] using old fashioned computer algebra platforms but the growing popularity of graphical platforms as Maple and Mathematica forced us to adapt and reconsider our methods and programs. We will describe some simple algebraic programming procedures (in Maple with GrTensorII package) for obtaining and the study of some exact solutions of the Einstein equations in order to convince a dedicated student in general relativity about the utility of a computer algebra system.
\end{abstract}

\section{Introduction}

Computational physics it is today recognized as a new branch of physics and. A branch of the computational physics I the computational relativity i.e. doing general relativity on the computer. Usualy computational relativity includes both algebraic computing (i.e. manipulation of mathematical expressions as tensors, polynomials, functions, etc. in symbolic form) and numerical computation (i.e. numerical simulations with/of Einstein equations in concrete systems). The main reason for the use of computer facilities in general relativity [2] is related to the complex structure of the Einstein equations (10 second order nonlinear partial differential equations) which impose long, time consuming, cumbersome and not straightforward computing manipulations. The development of computer science with advanced computing platforms (as Maple is) done possible both types of computation (symbolic and numerical) to do in general relativity for a large spectra of applications.

On the other side of the story, teaching students (even graduates) general relativity could be a difficult task, mainly because of the huge mathematical formalism necessary (differential geometry, tensorial algebra etc.). This make students reluctant to enter in such an adventure. Some years ago we reported [1] our experience in teaching undergraduate students 
general relativity using REDUCE platform. Unfortunately, our good results and experience was not possible to extend, because REDUCE is no more on the market (if still exist it has a primitive graphical interface and restricted facilities to do high level numerical computations (simulations, animations, etc.). Maple platform [9] becomes more popular - actually we teach in the computational physics lab our students Maple and its use for physics. As a consequence we had to adapt our methods in teaching general relativity with Maple - of course using also GrTensorII package, a free software embedded in Maple for use in Riemannian geometry, general relativity and cosmology [10]. The article is dedicated to these facts and is organized as follows : the next Section no. 2 describes briefly the Maple and GrTensorII facilities while the Section no. 3 describes the program and its use for finding a well-known solution of Einstein equations, namely the Schwarzschild solution. The Section no. 4 reviews the use of Maple + GrTensorII in cosmology, without great details (reported elsewhere [4-6]). A last section is dedicated to some conclusions.

\section{Maple+GrTensorII}

Maple is a general-purpose commercial computer algebra system. It was first developed in 1981 by the Symbolic Computation Group at the University of Waterloo in Waterloo, Ontario, Canada. Since 1988, it has been developed and sold commercially by Waterloo Maple Inc. (also known as Maplesoft), a Canadian company also based in Waterloo, Ontario. The current version is Maple 15. Maple is an interpreted, dynamically typed programming language [9]. As is usual with computer algebra systems, symbolic expressions are stored in memory as directed acyclic graphs. Since Maple 6 the language has permitted variables of lexical scope.

GrTensor II is a computer algebra package [10] for performing calculations in the general area of differential geometry. Its purpose is the calculation of tensor components on curved spacetimes specified in terms of a metric or set of basis vectors. Though originally designed for use in the field of general relativity, GRTensorII is useful in many other fields.

GRTensor II is not a stand alone package, but requires an algebraic engine. GRTensorII runs with all versions of Maple. GRTensor II and related software and documentation are distributed free of charge as an aide for both research and teaching; see at [10]. Authors of GrTensor are Peter Musgrave, Denis Pollney (!!!) and Kayll Lake.

The geometrical environment of GrTensorII is a Riemannian manifold with connection compatible with the riemannian metric. Special commands and routines for calculating geometrical objects as :

- the metric and the line element ( qload(), g(dn,dn),,makeg() )

- Chrisstoffel symbols and the covariant derivative (Chr(up,dn,dn)) 
- Ricci tensor and Ricci scalar (R(dn,dn), Ricciscalar)

- Einstein tensor (G(up,dn)), etc.

Other facilities are available, as : several predefined metrics and possibiliy to define new ones, manipulating with indices, extracting tensor components, defining new tensors using their natural definitions, advanced simplification routines...

An example easy to process is calculating and checking the Bianchi identities , namely $\boldsymbol{G}^{i}{ }_{j ; i}=\mathbf{O}$ where $G_{i j}=R_{i j}-\frac{1}{2} g_{i j} R$ is the Einstein tensor and “;” denotes the covariant derivative, $g_{i j}$ is the metric tensor. $R_{i j}$ and $R$ the Ricci tensor and Ricci scalar respectively. Thus we have a simple sequence of Maple+ GrTensorII commands :

$>$ grtw(); - it starts GrTensorII

$>$ qload(rob_sons); - loads Robertson Walker metric [3] previously defined

$\left.>\operatorname{grdef}\left(\mathbf{b i a}^{\wedge}{ }^{\wedge} \mathbf{i}\right\}:=\mathbf{G}\left\{\wedge^{\wedge} \mathbf{i} \wedge^{\wedge} \mathbf{j} ; \mathbf{j}\right\}\right) ;$ - defines above Biabchi object

> grcalc(bia(up)); gralter(bia(up),simplify); - calculates and simplifies Binachi object

$>$ grdisplay(bia(up));

If the metric is compatible with the riemannian connection the components of the Bianchi tensor must vanish. For the Robertson-Walker metric above (called "rob_sons") it gives:

\section{For the rob_sons spacetime: bi a(up) \\ bi $a(u p)=$ All components are zero}

\section{Finding an exact solution - the Schwarzschild metric}

This a metric describing the gravity around a pointlike mass $\mathrm{m}$

$$
d s^{2}=\left(1-\frac{2 m}{r}\right) d t^{2}-\frac{1}{1-\frac{2 m}{r}} d r^{2}-r^{2}\left(d \theta^{2}+\sin ^{2} \theta d \varphi^{2}\right)
$$

in spherical coordinates and in geometrical units $\mathrm{c}=\mathrm{G}=1$. It is a static, spherical symmetric and isotropic metric, and there are two singularities for $\mathrm{r}=0$ and $\mathrm{r}=\mathrm{m}$. The Schwarzschild metric is an exact solution of the Einstein equations. It is the most famous exact solution of Einstein equations - actually ot the vacuum Einstein equations, $R_{i j}=0$ valuable outside the point $\mathrm{r}=0$ where we don't have any matter $\left(\mathrm{T}_{\mathrm{ij}}=0\right)$. Today it is intensively used to describe a black-holes or other object of this type.

This example can be used to teach the students Einstein equations and their exact solutions and Maple+GrTensorII manipulation. How we done this? 
First we predefine (with makeq command) a static, spherical symmetric and isotropic metric ("sfomiz") having the shape as:

$$
d s^{2}=e^{2 \lambda(r)} d t^{2}-e^{2 v(r)} d r^{2}-r^{2}\left(d \theta^{2}+\sin ^{2} \theta d \varphi^{2}\right)
$$

Where we have two unknown functions of the radial coordinate, (Lr) and $N(r)$. Then we solve the Einstein equation with a sequence of maximum 25 command lines. Lets do this directly in a Maple worksheet as:

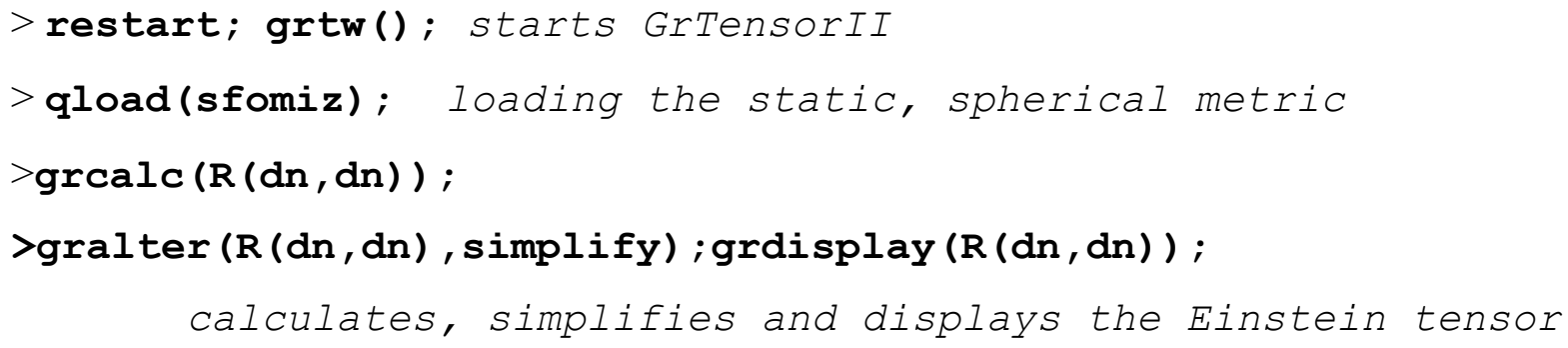

The next sequence of commands extractats the 4 Einstein equations from Einstein tensor obtaining 4 Maple objects, followed by a series of simplifications and substitutions commanda usually in Maple manipulations :

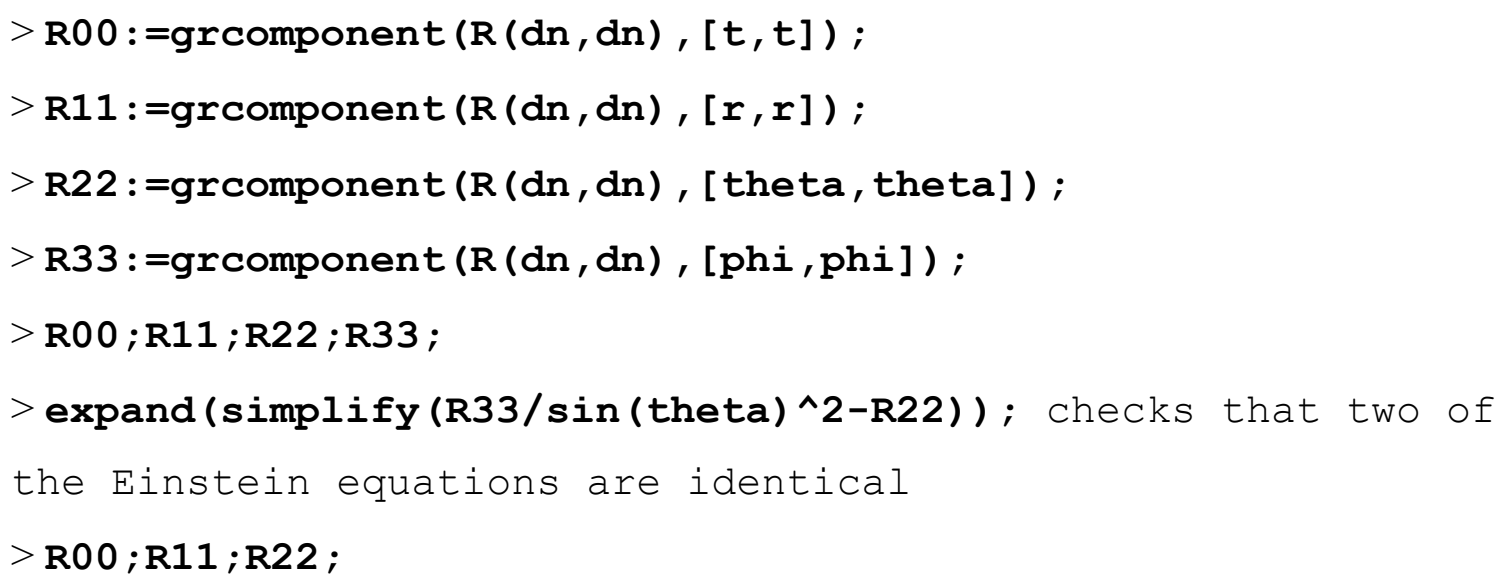

Now we solve the first equation in terms of 1 " (the second derivative in terms of the radial coordinate $r$ ) then check if the solution is good:

$>$ cccc: =solve (subs (diff (lambda $(r), r, r)=\operatorname{cccc}, R 00), \operatorname{cccc})$;

$>$ expand (simplify (subs $(\operatorname{diff}(\operatorname{lambda}(r), r, r)=\operatorname{cccc}, R 00)))$;

$$
-\frac{\left(\frac{d}{d r} \lambda(r)\right)\left(-r\left(\frac{d}{d r} v(r)\right)+r\left(\frac{d}{d r} \lambda(r)\right)+2\right)}{r}
$$

Now we have two differential equations for the the two unknown functions (after substituting the above expression for cccc) :

>ecul :=expand (simplify (subs (diff $(\operatorname{lambda}(r), r, r)=\operatorname{cccc}, R 11 * r / 2))$ ); 
$>\operatorname{ecu} 2:=\operatorname{expand}\left(\operatorname{simplify}\left(\mathrm{R} 22 * \exp (\mathrm{nu}(\mathrm{r}))^{\wedge} 2\right)\right)$;

ecul; ecu2 ;

$$
\begin{gathered}
\left(\frac{d}{d r} \lambda(r)\right)+\left(\frac{d}{d r} v(r)\right) \\
-r\left(\frac{d}{d r} \lambda(r)\right)+r\left(\frac{d}{d r} v(r)\right)+\left(\mathbf{e}^{v(r)}\right)^{2}-1
\end{gathered}
$$

We now solve the first of the above equations in terms of the $\mathrm{L}^{\prime}$

$>$ bbbb:=solve (subs (diff (lambda $(r), r)=b b b b, e c u 1), b b b b)$;

$>\operatorname{simplify}(\operatorname{expand}(\operatorname{subs}(\operatorname{diff}(\operatorname{lambda}(r), r)=b b b b$, ecu1) ) ) ;

Finally we have one single differential equation for $n(r)$ which we will solve it with dsolve [9] command :

> ecuatia:=simplify (expand ( $\operatorname{subs}(\operatorname{diff}(\operatorname{lambda}(r), r)=b b b b$, ecu2 $)))$;

$>$ dsolve (ecuatia, nu ( $r)$ ) ;

$$
\frac{1}{2} \ln \left(\frac{r}{-1+r \mathbf{e}^{-C 1}}\right)+\frac{C 1}{2}
$$

This finally gives (with a proper choose of the constants in order to fit with the newtonian limit - see [2]) the two unknown functions and the above Schwarzschild solution.

\section{Cosmology using Maple and GrTensorII}

A very popular topic among modern students is cosmology $[2,3]$ and its implications in the knowledge of the behavior of the Universe since Big-Bang till today when astrophysicists discovered the accelerated expansion of the actual universe $[7,8]$ called cosmic acceleration. Actually cosmology is one of the most active topics of modern physics, the cosmic acceleration not fitting with the Standard model of the universe.

Anyway the study of the Standard model is still a good topic for students. As it is known it starts with the Einstein equations written for a homogeneous, isotropic and only time depending metric called Friedman-Robertson-Walker:

$$
d s^{2}=-c^{2} d t^{2}+R(t)^{2}\left[\frac{d r^{2}}{1-k r^{2}}+r^{2}\left(d \theta^{2}+\sin ^{2}(\theta) d \varphi^{2}\right)\right]
$$

where $\mathrm{c}$ is the speed of light and $\mathrm{R}(\mathrm{t})$ is called scale factor. As a result the Einstein equations can be cast as Friedman equations if we assume the matter in the Universe as an isotropic homogeneous fluid matter (of all kind) - in the stress energy tensor. These are perfect issues for the use of a computer algebra program (of course in Maple and with GrTensorII) and we have an entire library for these purposes (Cosmo library) in all detaile described in a 
series of articles published by our group [4-6]. This library is currently used in our lectures on cosmology and of course for research purposes, because the library can be developed for certain more complicated situations, as a scalar field minimally or non-minimally coupled with gravity, higher order gravity theories and so on.

\section{Conclusions}

We already had more than ten years of experience in teaching general relativity and cosmology in the computer lab using computer algebra codes. Of course this cannot replace the teaching of the physics which general relativity implies, but can speed up the understanding and the use of the main results and concepts of this beautiful and elegant theory. On the other side it can open an easier door to research in the field and some of our students already followed this way, with good and striking results.

\section{Acknowledgements}

This work was supported by the grant POSDRU 107/1.5/G/13798, inside POSDRU Romania 2007-2013 co-financed by the European Social Fund - Investing in People. This article is mainly based on a talk given by D.N.V. at the $12^{\text {th }}$ International Balkan workshop on Applied Physics, July 6-8. Constanţa, România and a series of talks given at the University of Niş, Serbia. The kind and worm hospitality D.N.V. experienced in Nis and the support of the SEENET-MTP network is also acknowledged

\section{References}

[1] F. A. Ghergu, D. N. Vulcanov - Computing in Science and Engineering, 13 (2001) 65;

[2] C. W. Misner, K. S. Thorne, J. A. Wheeler, Gravitation, Freeman, San Francisco (1973);

[3] J. N. Islam, An Introduction to Mathematical Cosmology, Cambridge University Press, Cambridge (1992);

[4] D. N. Vulcanov, V. D. Vulcanov, Analele Univ. de Vest din Timisoara, Seria Matematica-Informatica, vol. XLII, Fasc. special 2 (2004) 181; electronic version at arXiv:cs/0409006;

[5] D. N. Vulcanov, IEEE Computer Society SYNASC06 ConferencePost-proceedings (2006) 47, electronic version at IEEE Digital Library and arXiv:gr-qc/0210006;

[6] D. N. Vulcanov, Central Europ. J. of Physics, 6 (2008) 84;

[7] J. P. Ostriker, P. J. Steinhardt, Nature, 377 (1995) 600;

[8] S. Perlmutter et.al., Astrophys. J, 517 (1999) 565;

[9] K. H. Heal, M. L. Hansen, K. M. Richard, Maple6 -Learning guide. Waterloo Maple Inc. (2000);

[10] http://grtensor.org. 